PoS $\quad \begin{aligned} & \text { PROCEEDINGS } \\ & \text { OF SCIENCE }\end{aligned}$

\title{
Production of lepton, quark and meson pairs in peripheral ultrarelativistic heavy ion collisions
}

\section{Antoni SZCZUREK*}

Rzeszow University, Poland

Institute of Nuclear Physics, Poland

E-mail: antoni.szczurek@ifj.edu.pl

We briefly review our results on exclusive production of $\mu^{+} \mu^{-}$, heavy quark-antiquark, and pairs of mesons in ultraperipheral, ultrarelativistic heavy ion collisions.

The 2011 Europhysics Conference on High Energy Physics - HEP 2011

July 21-27, 2011

Grenoble, Rhône-Alpes, France

${ }^{*}$ Speaker. 


\section{Introduction}

Ultrarelativistic collisions of heavy ions provide a nice oportunity to study photon-photon collisions [1]. In this type of reactions virtual (almost real) photons couple to the nucleus as a whole. Naively one expects an enhancement of the cross section proportional to $Z_{1}^{2} Z_{2}^{2}$ which is a huge factor. Recently we have shown that the inclusion of realistic charge distributions and realistic nucleus charge form factor makes the cross section smaller than the naive predictions. Several processes have been discussed in the literature. Recently we have studied some of them.

We have discussed production of $\mu^{+} \mu^{-}$pairs [2] heavy-quark heavy-antiquark pairs [3] as well as production of two mesons: $\rho^{0} \rho^{0}$ pairs [4], $\pi^{+} \pi^{-}$pairs [5] as well as of $D \bar{D}$ meson pairs [6].

\section{Formalism}

The equivalent photon approximation (EPA) is a standard alternative to the Feynman rules for calculating cross sections of electromagnetic interactions [7]. Due to the coherent action of all protons in the nucleus, the electromagnetic field surrounding the ions is very strong. This field can be viewed as a cloud of virtual photons. In the collision of two ions, these quasireal photons can collide with each other and with the other nucleus. In order to calculate the cross section of a process it is convenient to introduce: $x=\omega / E_{A}$, where $\omega$ energy of the photon and the energy of the nucleus and $E_{A}=\gamma A m_{\text {proton }}=\gamma M_{A}$ and $M_{A}$ is the mass of the nucleus and $E_{A}$ is the energy of the nucleus.

Below we consider a generic reaction $A A \rightarrow A A c_{1} c_{2}$. In the EPA the total cross section is calculated by the convolution:

$$
\sigma\left(A A \rightarrow c_{1} c_{2} A A ; s_{A A}\right)=\int \hat{\sigma}\left(\gamma \gamma \rightarrow c_{1} c_{2} ; W_{\gamma \gamma}=\sqrt{x_{1} x_{2} s_{A A}}\right) \mathrm{d} n_{\gamma \gamma}\left(x_{1}, x_{2}, \mathbf{b}\right) .
$$

The luminosity function $d n_{\gamma \gamma}$ above can be expressed in term of flux factors of photons prescribed to each of the nucleus:

$$
\mathrm{d} n_{\gamma \gamma}\left(\omega_{1}, \omega_{2}, \mathbf{b}\right)=\int S_{a b s}^{2}(\mathbf{b}) \mathrm{d}^{2} \mathbf{b}_{1} N\left(\omega_{1}, \mathbf{b}_{1}\right) \mathrm{d}^{2} \mathbf{b}_{2} N\left(\omega_{2}, \mathbf{b}_{2}\right) \frac{\mathrm{d} \omega_{1}}{\omega_{1}} \frac{\mathrm{d} \omega_{2}}{\omega_{2}} .
$$

The presence of the absorption factor $S_{a b s}^{2}(\mathbf{b})$ assures that we consider only peripheral collisions, when the nuclei do not touch each other. This can be taken into account by the following approximation:

$$
S_{a b s}^{2}(\mathbf{b})=\theta\left(\mathbf{b}-2 R_{A}\right)=\theta\left(\left|\mathbf{b}_{1}-\mathbf{b}_{2}\right|-2 R_{A}\right) .
$$

In the present case, we concentrate on processes with final nuclei in the ground state.

The charge distribution obtained from those experiments is often parametrized with the help of two-parameter Fermi model:

$$
\rho(r)=\rho_{0}\left(1+\exp \left(\frac{r-c}{a}\right)\right)^{-1}
$$

where $c$ is the radius of the nucleus, $a$ is the so-called diffuseness parameter of the charge density. 
Mathematically the charge form factor is the Fourier transform of the charge distribution:

$$
F(q)=\int \frac{4 \pi}{q} \rho(r) \sin (q r) r d r
$$

The monopole form factor [8] $F\left(q^{2}\right)=\frac{\Lambda^{2}}{\Lambda^{2}+q^{2}}$ leads to a simplification of many formulae for production of pairs of particles via photon-photon subprocess in nucleus-nucleus collisions. In our calculation $\Lambda$ is adjusted to reproduce root mean square radius $\Lambda=\sqrt{\frac{6}{\left.<r^{2}\right\rangle}}$.

\section{Results}

\subsection{Exclusive production of $\mu^{+} \mu^{-}$pairs}

Elementary cross section for charged leptons can be calculated within Quantum Electrodynamics. Several groups have made relevant calculations (see e.g. [9, 10, 11, 12]).

In Ref.[2] we have presented several distributions in muon rapidity and transverse momentum for RHIC and LHC experiments, including experimental acceptances. We have demonstrated how important is inclusion of realistic form factor in order to obtain realistic distributions of muons.
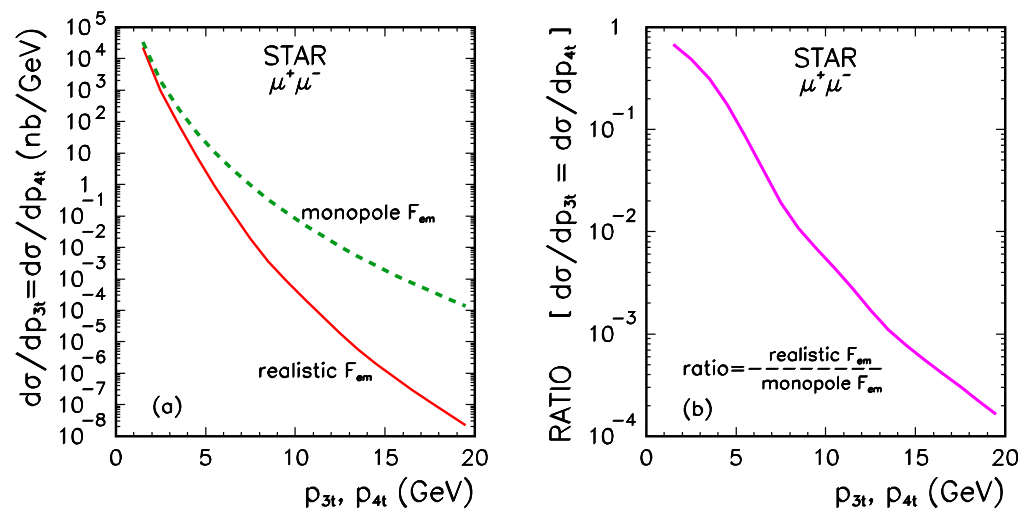

Figure 1: $\frac{\mathrm{d} \sigma}{\mathrm{d} p_{3 t}}$ (left) and the ratio (right) for the STAR conditions: $y_{3}, y_{4} \in(-1,1), p_{3 t}, p_{4 t} \geq 1 \mathrm{GeV}$ and $W_{N N}=200 \mathrm{GeV}$.

The distribution in the muon transverse momentum for STAR detector is shown in Fig.1. The STAR rapidity cuts $-1<y_{3}, y_{4}<1$ are taken here into account. The inclusion of realistic charge distribution is here extremely important. The relative effect of damping of the cross section with respect to the results with the monopole charge form factor is shown in the right panel. At $p_{t}=10$ $\mathrm{GeV}$ the damping factor is as big as 100 !

The ALICE collaboration can measure only forward muons with psudorapidity $3<\eta<4$ and has relatively low cut on muon transverse momentum $p_{t}>2 \mathrm{GeV}$. In Fig.2 (left panel) we show invariant mass distribution of dimuons for monopole and realistic form factors including the ALICE cuts. The bigger invariant mass, the bigger the difference between the two results. The same is true for distributions in muon transverse momenta (see the right panel). 

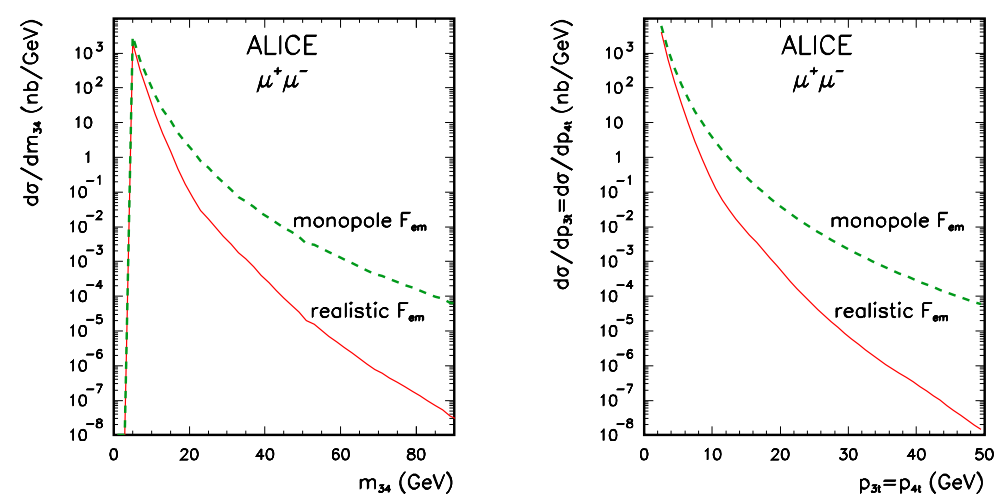

Figure 2: $\frac{\mathrm{d} \sigma}{\mathrm{d} W_{\gamma \gamma}}$ (left) and $\frac{\mathrm{d} \sigma}{\mathrm{d} p_{3 t}}=\frac{\mathrm{d} \sigma}{\mathrm{d} p_{4 t}}$ (right) for ALICE conditions: $y_{3}, y_{4}=(3,4), p_{3 t}, p_{4 t} \geq 2 \mathrm{GeV}$.

\subsection{Exclusive production of $c \bar{c}$ and $b \bar{b}$}

In Ref.[3] we have discussed several photon-photon processes leading to the $Q \bar{Q}$ in the final state.

Let us start with the Born direct contribution. The leading-order elementary cross section for $\gamma \gamma \rightarrow Q \bar{Q}$ as a function of $W_{\gamma \gamma}$ takes a simple form which differs from that for $\gamma \gamma \rightarrow l^{+} l^{-}$by color factors and fractional charges of quarks. In the current calculation we take: $m_{c}=1.5 \mathrm{GeV}$, $m_{b}=4.75 \mathrm{GeV}$. It is obvious that the final $Q \bar{Q}$ state cannot be observed experimentally due to the quark confinement and rather heavy mesons have to be observed instead. Presence of additional few light mesons is rather natural. This forces one to include also more complicated partonic final states.

In contrast to QED production of lepton pairs, in the case of $Q \bar{Q}$ production one needs to include also higher-order QCD processes. Here we include leading-order corrections only for the direct contribution. In $\alpha_{s}$-order there occur one-gluon bremsstrahlung diagrams $(\gamma \gamma \rightarrow Q \bar{Q} g)$ and interferences of the Born diagram with self-energy diagrams (in $\gamma \gamma \rightarrow Q \bar{Q}$ ) and vertex-correction diagrams (in $\gamma \gamma \rightarrow Q \bar{Q}$ ). We have followed the approach presented in Ref. [13].

We include also the subprocess $\gamma \gamma \rightarrow Q \bar{Q} q \bar{q}$, where $q(\bar{q})$ are $u, d, s$, quarks (antiquarks). The cross section for this mechanism can be calculated in the color dipole framework [14, 15]. In the dipole-dipole approach [15] the total cross section for the $\gamma \gamma \rightarrow Q \bar{Q}$ production reads:

$$
\begin{aligned}
& \sigma_{\gamma \gamma \rightarrow Q \bar{Q}}^{4 q}\left(W_{\gamma \gamma}\right) \\
& =\sum_{f_{2} \neq Q} \int\left|\Phi^{Q \bar{Q}}\left(\rho_{1}, z_{1}\right)\right|^{2}\left|\Phi^{f_{2} \bar{f}_{2}}\left(\rho_{2}, z_{2}\right)\right|^{2} \sigma_{d d}\left(\rho_{1}, \rho_{2}, x_{Q f}\right) d^{2} \rho_{1} d z_{1} d^{2} \rho_{2} d z_{2} \\
& +\sum_{f_{1} \neq Q} \int\left|\Phi^{f_{1} \bar{f}_{1}}\left(\rho_{1}, z_{1}\right)\right|^{2}\left|\Phi^{Q \bar{Q}}\left(\rho_{2}, z_{2}\right)\right|^{2} \sigma_{d d}\left(\rho_{1}, \rho_{2}, x_{f Q}\right) d^{2} \rho_{1} d z_{1} d^{2} \rho_{2} d z_{2},
\end{aligned}
$$

where $\Phi^{Q \bar{Q}}(\rho, z)$ are the quark - antiquark wave functions of the photon in the mixed representation and $\sigma_{d d}$ is the dipole-dipole cross section. Eq.(3.1) is correct at sufficiently high energy $W_{\gamma \gamma} \gg$ $2 m_{Q}$. At lower energies, the proximity of the kinematical threshold must be taken into account. 
The cross section for the $\gamma \gamma \rightarrow Q \bar{Q} q \bar{q}$ process here is much bigger than the one corresponding to the tree-level Feynman diagram as it effectively resums higher-order QCD contributions.

As discussed in Ref. [15] the $Q \bar{Q} q \bar{q}$ component have very small overlap with the singleresolved component because of quite different final state. The cross section for the single-resolved contribution can be written as:

$$
\sigma_{1-r e s}(s)=\int d x_{1}\left[g_{1}\left(x_{1}, \mu^{2}\right) \hat{\sigma}_{g \gamma}\left(\hat{s}=x_{1} s\right)\right]+\int d x_{2}\left[g_{2}\left(x_{2}, \mu^{2}\right) \hat{\sigma}_{\gamma g}\left(\hat{s}=x_{2} s\right)\right],
$$

where $g_{1}$ and $g_{2}$ are gluon distributions in photon 1 or photon 2 and $\hat{\sigma}_{q \gamma}$ and $\hat{\sigma}_{\gamma g}$ are elementary cross sections. In our calculation we take the gluon distribution from Ref. [16].

Elementary cross sections have been presented and discussed in Ref.[3]. Here we show only nuclear cross sections. In Fig. 3 we compare the contributions of the different mechanisms as a function of the photon-photon subsystem energy. For the Born case it is identical as a distribution in quark-antiquark invariant mass. These distributions reflect the energy dependence of the elementary cross sections. Please note a sizable contribution of the leading-order corrections close to the threshold and at large energies for the $c \bar{c}$ case. Since in this case $W_{\gamma \gamma}>M_{Q \bar{Q}}$, it becomes clear that the $Q \bar{Q} q \bar{q}$ contributions must have much steeper dependence on the $Q \bar{Q}$ invariant mass than the direct one which means that large $Q \bar{Q}$ invariant masses are produced mostly in the direct process. In contrast, small invariant masses are populated dominantly by the four-quark contribution.
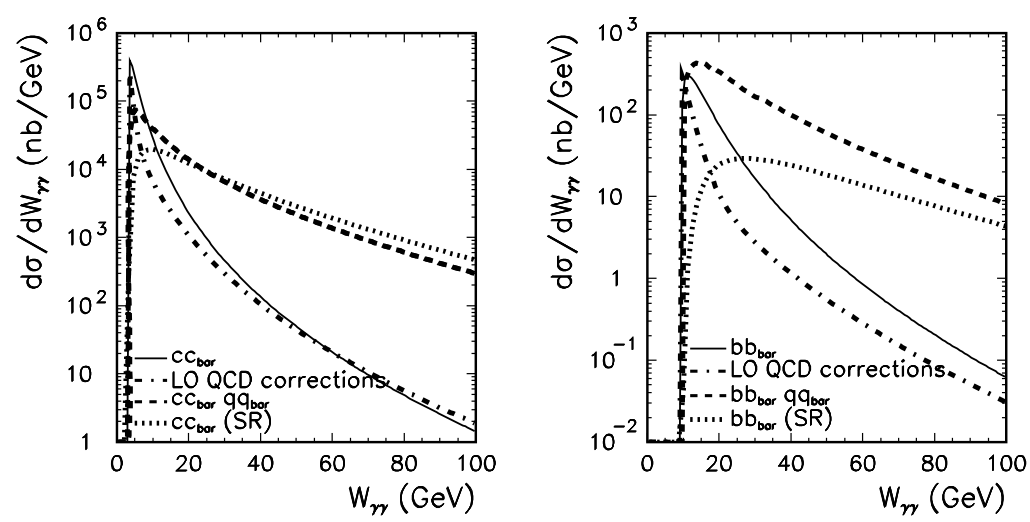

Figure 3: The nuclear cross section as a function of photon-photon subsystem energy $W_{\gamma \gamma}$ in EPA. The solid line denotes the results corresponding to the Born amplitude ( $c \bar{c}$-left panel and $b \bar{b}$-right panel). The leading-order QCD corrections are shown by the dash-dotted line. For comparison we show the differential distributions in the case when an additional pair of light quarks is produced in the final state (dashed lines) and for the single-resolved components (dotted line).

\subsection{Exclusive production of $\pi^{+} \pi^{-}$pairs}

Here we discuss production of "large" invariant mass $\pi^{+} \pi^{-}$pairs. Brodsky and Lepage developed a relevant perturbative formalism [17]. The invariant amplitude for the initial helicities of two photons can be written as the following convolution:

$$
\mathscr{M}\left(\lambda_{1}, \lambda_{2}\right)=\int_{0}^{1} d x \int_{0}^{1} d y \phi_{\pi}\left(x, \mu_{x}^{2}\right) T_{H}^{\lambda_{1} \lambda_{2}}\left(x, y, \mu^{2}\right) \phi_{\pi}\left(y, \mu_{y}^{2}\right) .
$$


We take the helicity dependent hard scattering amplitudes from Ref. [18]. These scattering amplitudes are different for $\pi^{+} \pi^{-}$and $\pi^{0} \pi^{0}$. The distribution amplitudes are subjected to the ERBL pQCD evolution $[19,20]$.

Different distribution amplitudes have been used in the past. Recently Wu and Huang [21] proposed a new distribution amplitude based on a light-cone wave function.

The pion distribution amplitude at the initial scale is controlled by a parameter B. They have found that the BABAR data for pion transition form factor at low and high transferred fourmomentum squared regions can be described by setting $B \approx 0.6$. In the following we shall use $B=0.6$ and $m_{q}=0.3 \mathrm{GeV}$. Then $A=16.62 \mathrm{GeV}^{-1}$ and $\beta=0.745 \mathrm{GeV}$.

The hand-bag model was proposed as an alternative for the leading term Brodsky-Lepage pQCD approach [22]. As in the case of BL pQCD the hand-bag approach applies at large Mandelstam variables $s \sim-t \sim-u$ i.e. at large momentum transfers. In this approach the ratio of the cross section for the $\pi^{0} \pi^{0}$ process to that for the $\pi^{+} \pi^{-}$process does not depend on $\theta$ and is $\frac{1}{2}$. The nonperturbative object $R_{\pi \pi}(s)$ in the hand-bag amplitude, describing transition from a quark pair to a meson pair, cannot be calulated from first principles. In Ref. [22] the form factor was parametrized in terms of the valence and non-valence form factors as:

$$
R_{\pi \pi}(s)=\frac{5}{9 s} a_{u}\left(\frac{s_{0}}{s}\right)^{n_{u}}+\frac{1}{9 s} a_{s}\left(\frac{s_{0}}{s}\right)^{n_{s}} .
$$

The $a_{u}, n_{u}, a_{s}$ and $n_{s}$ values found from the fit in Ref. [22] slightly depend on energy. For simplicity we have averaged these values and used: $a_{u}=1.375 \mathrm{GeV}^{2}, n_{u}=0.4175, a_{s}=0.5025 \mathrm{GeV}^{2}$ and $n_{s}=1.195$.

In Fig. 4 we show distribution in the two-pion invariant mass which by the energy conservation is also the photon-photon subsystem energy. Here we have taken experimental limitations usually used for the $\pi \pi$ production in $e^{+} e^{-}$collisions. In the same figure we show our results for the $\gamma \gamma$ collisions extracted from the $e^{+} e^{-}$collisions together with the corresponding nuclear cross sections for $\pi^{+} \pi^{-}$(left panel) and $\pi^{0} \pi^{0}$ (right panel) production.

Comparing the elementary and nuclear cross sections we see a large enhancement of the order of $10^{4}$ which is somewhat less than $Z_{1}^{2} Z_{2}^{2}$ one could expect from a naive counting.

\subsection{Exclusive production of $\rho^{0} \rho^{0}$ pairs}

At low energies one observes a huge enhancement of the cross section for the elementary process $\gamma \gamma \rightarrow \rho^{0} \rho^{0}[4]$.

In Fig. 5 we show distribution in $\rho^{0} \rho^{0}$ invariant mass (left panel) and the ratio of the cross section for realistic and monopole form factors.

\subsection{Outlook}

We have presented selected examples of processes that could be studied at RHIC or LHC. In all cases we have obtained sizeable cross sections. We have shown that the inclusion of realistic charge form factor is necessary to obtain realistic particle distributions.

Measurements of the processes discussed here are not easy as one has to assure exclusivity of the process, i.e., it must be checked that there are no other particles than that measured in central detectors. In all cases fissibilty studies, including Monte Carlo simulations, are required. 

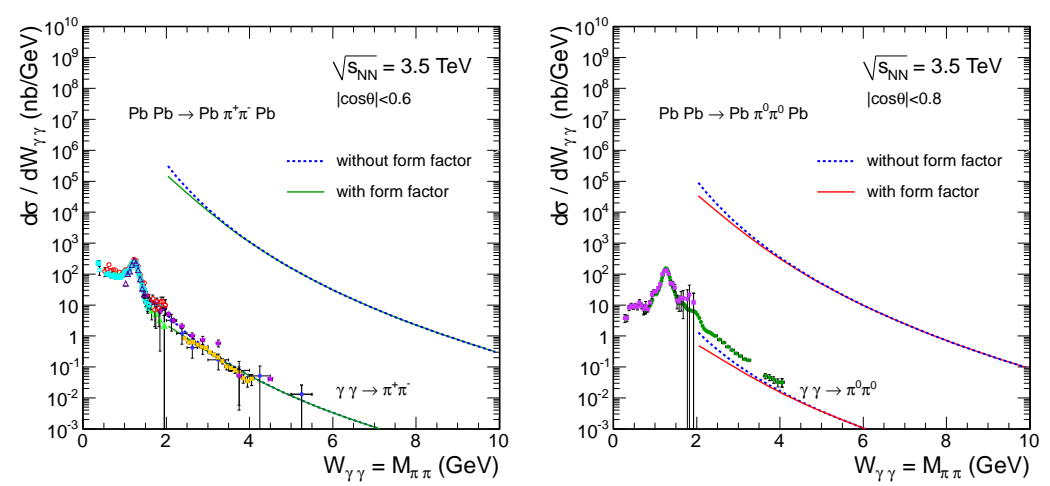

Figure 4: The nuclear (upper lines) and elementary (lower lines) cross section as a function of photonphoton subsystem energy $W_{\gamma \gamma}$ in the b-space EPA within the BL pQCD approach for the elementary cross section with $\mathrm{Wu}-\mathrm{Huang}$ distribution amplitude. The angular ranges in the figure caption correspond to experimental cuts.
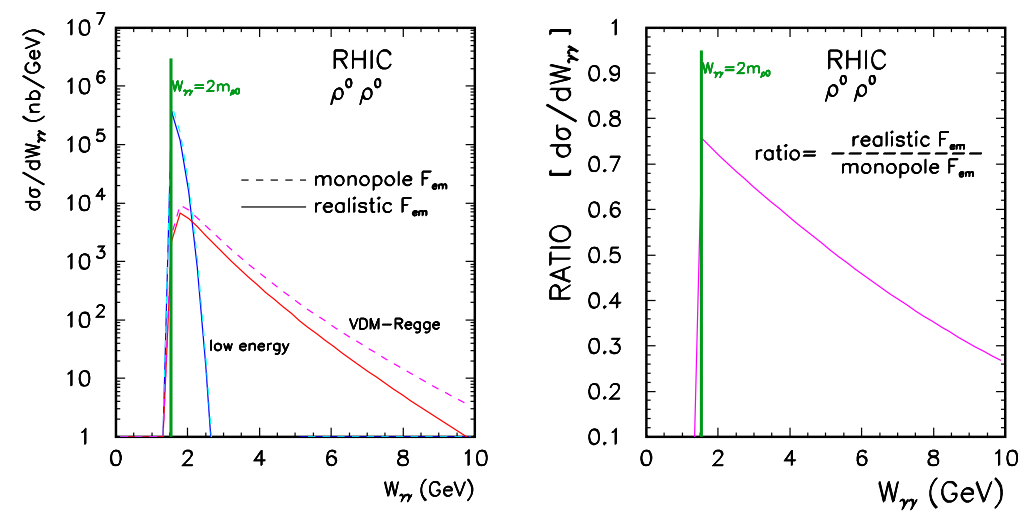

Figure 5: Distribution in the $\rho^{0} \rho^{0}$ invariant mass

I am indebted to Mariola Kłusek, Wolfgang Schäfer, Valerij Serbo and Magno Machado for collaboration on the topics presented here. I congratulate Johann Collot and collaborators for very good organization and friendly atmosphere during EPS2011.

\section{References}

[1] V.M. Budneev et al. Phys. Rep. 15 (1975) 4;

C.A. Bertulani, G. Baur, Phys. Rep. 163 (1988) 299;

G. Baur, K. Hencken, D. Trautmann, S. Sadovsky and Y. Kharlov, Phys. Rep. 364 (2002) 259;

C.A. Bertulani, S. Klein and J. Nystrand, J Ann. Rev. Nucl. Part. Sci.55 (2005) 271;

A. Baltz et al., Phys. Rep. 458 (2008) 1.

[2] M. Kłusek-Gawenda and A. Szczurek, Phys. Rev. C 82 (2010) 014904.

[3] M. Kłusek-Gawenda, A. Szczurek, M. Machado and V. Serbo, Phys. Rev. C 83 (2011) 024903.

[4] M. Kłusek, W. Schäfer and A. Szczurek, Phys. Lett. B 674 (2009) 92. 
[5] M. Kłusek-Gawenda and A. Szczurek, Phys. Lett. B 700 (2011) 322.

[6] M. Łuszczak and A. Szczurek, Phys. Lett. B 700 (2009) 116.

[7] J.D. Jackson, Classical Electrodynamics, 2nd ed. (Wiley, New York), 1975, p. 722.

[8] K. Hencken, D. Trautmann and G. Baur, Phys. Rev. A 49 (1994) 1584.

[9] G. Baur and C.A. Bertulani, Phys. Rev. C 35 (1987) 836.

[10] K. Hencken, D. Trautmann and G. Baur, Phys. Rev. C 59 (1999) 841.

[11] D.Yu. Ivanov, A. Schiller and V.G. Serbo, Phys. Lett. B 454 (1999) 155;

K. Henecken, E.A. Kuraev and V.G. Serbo, Phys. Rev. C 75 (2007) 034903;

U.D. Jentschura, K. Hencken and V.G. Serbo, Eur. Phys. J. C 58 (2008) 281;

U.D. Jentschura and V.G. Serbo, Eur. Phys. J. C 64 (2009) 309.

[12] A. Baltz, Phys. Rev. Lett. 100 (2008) 062302;

A. Baltz, Phys. Rev. C 80 (2009) 034901.

[13] B.A. Kniehl, A.V. Kotikov, Z.V. Merebashvili and O.L. Veretin, Phys. Rev. D 79 (2009) 114032.

[14] N. Timneanu, J. Kwieciński and L. Motyka, Eur. Phys. J. C 23 (2002) 513.

[15] A. Szczurek, Eur. Phys. J. C 26 (2002) 183.

[16] M. Gluck, E. Reya and A. Vogt, Phys. Rev. D 46 (1992) 1973.

[17] S.J. Brodsky and G.P. Lepage, Phys. Rev. D 24 (1981) 1808.

[18] C.R. Ji and F. Amiri, Phys. Rev. D 42 (1990) 3764.

[19] S.J. Brodsky and G.P. Lepage, Phys. Lett. B 87 (1979) 359.

[20] A.V. Efremov and A.V. Radyushkin, Phys. Lett. B 94 (1980) 245.

[21] X.G. Wu and T. Huang, Phys. Rev. D 82 (2010) 034024.

[22] M. Diehl, P. Kroll and C. Vogt, Phys. Lett. B 532 (2002) 99;

M. Diehl and P. Kroll, Phys. Lett. B 683 (2010) 165. 